\title{
LINEAR OPERATORS THAT PRESERVE GRAPHICAL PROPERTIES OF MATRICES: ISOLATION NUMBERS
}

\author{
LeRoy B. Beasley, Logan, Seok-Zun Song, Jeju, Young Bae Jun, Jinju
}

(Received July 18, 2013)

\begin{abstract}
Let $A$ be a Boolean $\{0,1\}$ matrix. The isolation number of $A$ is the maximum number of ones in $A$ such that no two are in any row or any column (that is they are independent), and no two are in a $2 \times 2$ submatrix of all ones. The isolation number of $A$ is a lower bound on the Boolean rank of $A$. A linear operator on the set of $m \times n$ Boolean matrices is a mapping which is additive and maps the zero matrix, $O$, to itself. A mapping strongly preserves a set, $S$, if it maps the set $S$ into the set $S$ and the complement of the set $S$ into the complement of the set $S$. We investigate linear operators that preserve the isolation number of Boolean matrices. Specifically, we show that $T$ is a Boolean linear operator that strongly preserves isolation number $k$ for any $1 \leqslant k \leqslant \min \{m, n\}$ if and only if there are fixed permutation matrices $P$ and $Q$ such that for $X \in \mathcal{M}_{m, n}(\mathbb{B}) T(X)=P X Q$ or, $m=n$ and $T(X)=P X^{t} Q$ where $X^{t}$ is the transpose of $X$.
\end{abstract}

Keywords: Boolean matrix; Boolean rank; Boolean linear operator; isolation number

MSC 2010: 15A86, 15A04, 15B34

\section{INTRODUCTION}

The binary Boolean algebra consists of the set $\mathbb{B}=\{0,1\}$ equipped with two binary operations, addition and multiplication. The operations are defined as usual except that $1+1=1$.

Matrices with entries in $\mathbb{B}$ have the same arithmetic as for real matrices except that the addition uses the Boolean sum. Matrices over more general semirings can be studied by considering their patterns of 0's and 1's. The graphical properties of matrices, those that are invariant under permutations of the rows and columns,

This research was supported by the Basic Science Research Program through the National Research Foundation of Korea (NRF) funded by the Ministry of Education, Science and Technology (No. 2012R1A1A2042193). 
usually are inherited from their patterns. Thus, we will limit our endeavors to matrices whose entries are only 0's and 1's.

There are many papers on linear operators on a matrix space that preserve matrix functions over an algebraic structure, see [2], [3], [4], [6] and [8]. Boolean matrices also have been the subject of research by many authors, see [4], [5], [7] and [8]. Beasley and Pullman [4] obtained characterizations of rank-preserving operators of Boolean matrices. Beasley, Kang and Song [6] characterized the linear operators that preserve term rank of matrices over the binary Boolean algebra and semirings.

In this article we consider the isolation number of a matrix, and use the fact that the isolation number is a lower bound on the Boolean rank to characterize the linear operators that preserve sets defined by the isolation number.

\section{Preliminaries}

Let $\mathcal{M}_{m, n}(\mathbb{B})$ be the set of all $m \times n$ matrices with entries in the binary Boolean algebra $\mathbb{B}$. The usual definitions for adding and multiplying matrices apply to Boolean matrices as well. The matrix $A^{(m, n)}$ denotes a matrix in $\mathcal{M}_{m, n}(\mathbb{B}), I_{n}$ is the $n \times n$ identity matrix, $O^{(m, n)}$ is the $m \times n$ zero matrix, and $J^{(m, n)}$ is the $m \times n$ matrix all of whose entries are 1 . Let $E_{i, j}^{(m, n)}$ be the $m \times n$ matrix whose $(i, j)^{\text {th }}$ entry is 1 and whose other entries are all 0 , then we call $E_{i, j}^{(m, n)}$ a cell. We will suppress the superscripts or subscripts on these matrices when the orders are evident from the context and we write $A, I, O, J$, and $E_{i j}$, respectively. For a matrix $A, \#(A)$ denotes the number of nonzero entries in $A$. Further, we let the set of all cells be denoted by $\mathcal{E}$. That is,

$$
\mathcal{E}=\left\{E_{i, j} \in \mathcal{M}_{m, n}(\mathbb{B}) ; i=1, \ldots, m \text { and } j=1, \ldots, n\right\}
$$

Boolean rank and isolation numbers. The Boolean rank, $\beta(A)$, of a nonzero Boolean matrix $A$ in $\mathcal{M}_{m, n}(\mathbb{B})$ is the minimal number $k$ such that there exist Boolean matrices $B \in \mathcal{M}_{m, k}(\mathbb{B})$ and $C \in \mathcal{M}_{k, n}(\mathbb{B})$ such that $A=B C$. The Boolean rank of the zero matrix is 0 . It is well known that $\beta(A)$ is the least $k$ such that $A$ is the sum of $k$ matrices of Boolean rank 1, see [4]. From now on we will assume that $2 \leqslant m \leqslant n$. It follows that $0 \leqslant \beta(A) \leqslant m$ for all nonzero $A \in \mathcal{M}_{m, n}(\mathbb{B})$.

By considering a minimal sum of rank one matrices for $A$ and $B$ such as $A=$ $A_{1}+\ldots+A_{k}$ and $B=B_{1}+\ldots+B_{l}$, we have that $A+B=A_{1}+\ldots+A_{k}+B_{1}+\ldots+B_{l}$, so that $A+B$ has rank at most $k+l$. This establishes the following lemma. 
Lemma 2.1. For matrices $A$ and $B$ in $\mathcal{M}_{m, n}(\mathbb{B})$, we have $\beta(A+B) \leqslant \beta(A)+\beta(B)$.

If $A$ and $B$ are matrices in $\mathcal{M}_{m, n}(\mathbb{B})$, we say that $B$ dominates $A$ (written $A \leqslant B$ or $B \geqslant A$ ) if $b_{i, j}=0$ implies $a_{i, j}=0$ for all $i$ and $j$. Equivalently, $A \leqslant B$ if and only if $A+B=B$. This provides a reflexive and transitive relation on $\mathcal{M}_{m, n}(\mathbb{B})$.

Let $A \in \mathcal{M}_{m, n}(\mathbb{B})$. A set, $\mathcal{I}_{N}(A)$, of indices is called a set of independent ones of $A$ if (1) the first coordinates are distinct, (2) the second coordinates are distinct and $(3)$ if $(i, j) \in \mathcal{I}_{N}(A)$, then $a_{i, j}=1$. A set, $\mathcal{I}_{S}(A)$, of independent ones is called a set of isolated ones of $A$ if for any pair, $(i, j),(k, l) \in \mathcal{I}_{S}(A)$, the submatrix of $A$ on rows $i$ and $k$ and on columns $j$ and $l$ is not $\left[\begin{array}{ll}1 & 1 \\ 1 & 1\end{array}\right]$. The isolation number of $A$, $\iota(A)$, is the maximum cardinality of any set of isolated ones in $A$.

In [1] it was shown that the set of matrices of Boolean rank one and the set of matrices whose isolation number is one are the same set. It was also shown that the set of matrices of Boolean rank two and the set of matrices whose isolation number is two are the same set.

Since no two isolated ones can lie in any single Boolean rank one submatrix, we have:

Lemma 2.2. Let $A \in \mathcal{M}_{m, n}(\mathbb{B})$. Then $\iota(A) \leqslant \beta(A)$.

However, as the following example shows, unless $\iota(A)=1,2$, or $\min (m, n)$ the only obvious relationship between isolation number and Boolean rank is that the isolation number never exceeds the Boolean rank of a matrix.

Example 2.3. Let $n \geqslant 3$ and let $D_{n}$ be the matrix $J \backslash I$, that is, $D_{n}$ is the matrix all of whose entries are ones except that all diagonal entries are zero. Then $\iota\left(D_{n}\right)=3$, for any $n \geqslant 3$. However, $\beta(A)=k$ where $k=\min \left\{k ; n \leqslant\left(\begin{array}{c}k \\ \lfloor k / 2\rfloor\end{array}\right)\right\}$, see [5]. So $\iota\left(D_{20}\right)=3$ while $\beta\left(D_{20}\right)=6$.

Let $A, B \in \mathcal{M}_{m, n}(\mathbb{B})$. Since those indices in any set of isolation numbers for $A+B$ whose corresponding entries in $A$ (or $B$ ) are ones in a set of isolation numbers for $A$ (or $B$, respectively), it follows:

Lemma 2.4. For matrices $A$ and $B$ in $\mathcal{M}_{m, n}(\mathbb{B})$, we have $\iota(A+B) \leqslant \iota(A)+\iota(B)$.

In [1] the structure of a matrix $A \in \mathcal{M}_{m, n}(\mathbb{B})$ whose isolation number is $\min \{m, n\}$ was provided. Thus, the set of matrices with isolation number $\min \{m, n\}$ can be systematically described, while the set of matrices of Boolean rank $\min \{m, n\}$ cannot.

Upper ideals. A subset, $\mathcal{U}$, of $\mathcal{M}_{m, n}(\mathbb{B})$ is called an upper ideal if $X \in \mathcal{U}$ implies $X+Y \in \mathcal{U}$ for every $Y \in \mathcal{M}_{m, n}(\mathbb{B})$. A set, $S$, is bigraphical if $X \in S$ 
implies $P X Q \in S$ for all permutation matrices $P$ and $Q$ of appropriate orders. The reason for this name is that the bipartite graph associated with a matrix has the same properties as any other bipartite that has only relabeling of the bipartite sets.

An upper ideal $\mathcal{U}$ is said to separate cells if for any two distinct cells $E$ and $F$ in $\mathcal{M}_{m, n}(\mathbb{B})$, there is some $X \notin \mathcal{U}$ such that $X+E \in \mathcal{U}$ while $X+F \notin \mathcal{U}$.

Let $\mathcal{F}$ be a subset of $\mathcal{M}_{m, n}(\mathbb{B})$. The upper ideal generated by $\mathcal{F}, \mathcal{U}(\mathcal{F})$, is the set of matrices not dominated by any element of $\mathcal{F}$, i.e., $\mathcal{U}(\mathcal{F})=\left\{A \in \mathcal{M}_{m, n}(\mathbb{B})\right.$; for all $\left.B \in \mathcal{M}_{m, n}(\mathbb{B}), A+B \notin \mathcal{F}\right\}$.

In [3] it was shown that if $\mathcal{F}$ is the set of Boolean rank $k$ matrices, $k \geqslant 2$, then $\mathcal{U}(\mathcal{F})$ separates cells.

Lemma 2.5. Let $\mathcal{F}$ be the set of matrices in $\mathcal{M}_{m, n}(\mathbb{B})$ whose isolation number is $k$, for some $2 \leqslant k \leqslant m$. Then $\mathcal{U}(\mathcal{F})$ separates cells.

Proof. Let $E$ and $F$ be cells in $\mathcal{M}_{m, n}(\mathbb{B})$. Then by permuting we can assume that $E=E_{1,1}$ and $F=E_{i, j}$ for some $(i, j) \neq(1,1)$. If $j \neq 1$ interchange the $j^{\text {th }}$ column with the $n^{\text {th }}$ column, so that $F=E_{i, n}$. If $j=1$ so that $i \neq 1$ interchange the $i^{\text {th }}$ row with the $m^{\text {th }}$ row, so that $F=E_{m, 1}$. In either case, since $k \leqslant \min \{m, n\}$, we have $F=E_{r, s}$ with $r+s \geqslant k+1$. Let $A=\left(a_{i, j}\right)$ where $a_{i, j}=0$ if $i+j \leqslant k$ and $a_{i, j}=1$ otherwise. Then $\{(i, j) ; i+j=k+1\}$ is a set of isolated ones of size $k$. Further, $A \geqslant F$ so $\iota(A+F)=k$ but $\iota(A+E)=k-1$. So $A+F \notin \mathcal{U}(\mathcal{F})$ while $A+E \in \mathcal{U}(\mathcal{F})$. That is, $\mathcal{U}(\mathcal{F})$ separates cells.

Boolean linear operators. A mapping $T: \mathcal{M}_{m, n}(\mathbb{B}) \rightarrow \mathcal{M}_{m, n}(\mathbb{B})$ is called a Boolean linear operator if for any $X, Y \in \mathcal{M}_{m, n}(\mathbb{B}), T(X+Y)=T(X)+T(Y)$, and $T(O)=O$.

Let $f: \mathcal{M}_{m, n}(\mathbb{B}) \rightarrow \mathbb{S}$ be a mapping where $\mathbb{S}$ is any set. Let $S$ be a subset of $\mathcal{M}_{m, n}(\mathbb{B})$. For a Boolean linear operator $T: \mathcal{M}_{m, n}(\mathbb{B}) \rightarrow \mathcal{M}_{m, n}(\mathbb{B})$, we say that $T$

(1) preserves $f$ if for any $k \in \mathbb{S}, f(T(X))=k$ whenever $f(X)=k$ for all $X \in$ $\mathcal{M}_{m, n}(\mathbb{B})$

(2) preserves $S$ if $T(X) \in S$ whenever $X \in S$ for all $X \in \mathcal{M}_{m, n}(\mathbb{B})$;

(3) strongly preserves $f$ if, for any $k \in \mathbb{S}, f(T(X))=k$ if and only if $f(X)=k$ for all $X \in \mathcal{M}_{m, n}(\mathbb{B})$

(4) strongly preserves $S$ if $T(X) \in S$ if and only if $X \in S$ for all $X \in \mathcal{M}_{m, n}(\mathbb{B})$.

A Boolean linear operator $T: \mathcal{M}_{m, n}(\mathbb{B}) \rightarrow \mathcal{M}_{m, n}(\mathbb{B})$ is called a $(P, Q)$-operator if there are permutation matrices $P$ and $Q$ such that $T(X)=P X Q$ for all $X \in$ $\mathcal{M}_{m, n}(\mathbb{B})$, or when $m=n, T(X)=P X^{t} Q$ for all $X \in \mathcal{M}_{m, n}(\mathbb{B})$, where $X^{t}$ is the transpose of $X$. 
A Boolean linear operator $T: \mathcal{M}_{m, n}(\mathbb{B}) \rightarrow \mathcal{M}_{m, n}(\mathbb{B})$ is said to be nonsingular if $T(X)=O$ only if $X=O$. Unlike linear operators over a field, nonsingular operators need not be invertible.

A line is a matrix of the form $R_{i}=\sum_{j=1}^{n} E_{i, j}$ or of the form $C_{j}=\sum_{i=1}^{m} E_{i, j}$. That is, a line is a matrix which includes all the ones in a row or column, and all other entries are 0.

In the following lemma we will use the notation: $R_{i}=\sum_{l=1}^{n} E_{i, l}$ is the $i^{\text {th }}$ full row matrix and $C_{j}=\sum_{k=1}^{m} E_{k, j}$ is the $j^{\text {th }}$ full column matrix.

Lemma 2.6. Let $T: \mathcal{M}_{m, n}(\mathbb{B}) \rightarrow \mathcal{M}_{m, n}(\mathbb{B})$ be a Boolean linear operator. If $T$ is bijective and maps lines to lines, then $T$ is a $(P, Q)$-operator.

Pr o of. Let $\mathcal{L}=\left\{R_{i} ; 1 \leqslant i \leqslant m\right\} \cup\left\{C_{j} ; 1 \leqslant j \leqslant n\right\}$. Then, since $T$ is bijective, $T$ is bijective on $\mathcal{L}$. If $m \neq n$, since $T$ is bijective, the image of each $R_{i}$ must be some $R_{k}$, and the image of each $C_{j}$ must be some $C_{l}$. This is easily seen by a counting argument.

If $m=n$ then either the image of every row is a row and hence the image of every column is a column, or the image of every row is a column and hence the image of every column is a row, since $T$ is bijective on $\mathcal{L}$. If the image of every row is a column and the image of every column is a row, composing $T$ with the transpose operator gives an operator that maps rows to rows and columns to columns. In these cases, letting $\sigma$ be a permutation such that $T\left(R_{i}\right)=R_{\sigma(i)}$ and $\tau$ be a permutation such that $T\left(C_{j}\right)=C_{\tau(j)}$, we have that $T$ is a $(P, Q)$-operator where $P$ is the permutation matrix corresponding to $\sigma$ and $Q$ is the permutation matrix corresponding to $\tau$.

Now, if $m=n$ and the image of one row is a row and the image of another row is a column, we say without loss of generality that $T\left(R_{1}\right)=R_{1}$ and $T\left(R_{2}\right)=C_{1}$. Then, $\#\left(R_{1}+R_{2}\right)=2 n$ while $\#\left(R_{1}+C_{1}\right)=2 n-1$, an impossibility since $T$ is bijective. Therefore $T$ is a $(P, Q)$-operator.

\section{Preservers of isolation number of MAtrices}

In [4] it was shown that if $T: \mathcal{M}_{m, n}(\mathbb{B}) \rightarrow \mathcal{M}_{m, n}(\mathbb{B})$ preserves Boolean ranks one and two, then $T$ is a $(P, Q)$-operator. Since the Boolean rank and isolation number of a matrix agree when their Boolean ranks are 1 and 2 we have the following theorem. 
Theorem 3.1. Let $T: \mathcal{M}_{m, n}(\mathbb{B}) \rightarrow \mathcal{M}_{m, n}(\mathbb{B})$ be a Boolean linear operator. Then the following assertions are equivalent:

(1) $T$ preserves the isolation number of matrices,

(2) $T$ preserves isolation numbers one and two,

(3) $T$ is a $(P, Q)$-operator.

Now, consider the Boolean linear operators that preserve upper ideals.

Lemma 3.2. Let $T: \mathcal{M}_{m, n}(\mathbb{B}) \rightarrow \mathcal{M}_{m, n}(\mathbb{B})$ be a Boolean linear operator. If an upper ideal $\mathcal{U}$ separates cells and $T$ strongly preserves $\mathcal{U}$ then $T$ is bijective on the set of cells.

P r o of. Since $\mathcal{M}_{m, n}(\mathbb{B})$ is finite, there is a power of $T$, say $T^{q}$ which is idempotent. Let $L=T^{q}$. Then $L$ strongly preserves $\mathcal{U}$ since $T$ does, and $L^{2}=L$.

Suppose that $T(X)=O$. If a cell $E \leqslant X$ then $T(E)=O$. Let $F$ be any other cell. Since $\mathcal{U}$ separates cells, there is some $N \in \mathcal{M}_{m, n}(\mathbb{B})$ which separates $F$ from $E$. That is, $N \notin \mathcal{U}, N+F \in \mathcal{U}$ but $N+E \notin \mathcal{U}$. But then $T(N)+T(E)=T(N) \leqslant T(N+F)$ and since $N+F \in \mathcal{U}, T(N)=T(N+F) \in \mathcal{U}$, contradicting that $T$ strongly preserves $\mathcal{U}$. Thus, $T$, and hence $L$, is nonsingular.

Now, let $E$ be any cell and suppose that $F \leqslant L(E)$. If $F \neq E$, let $N$ separate $F$ from $E$. That is, $N \notin \mathcal{U}, N+F \in \mathcal{U}$ but $N+E \notin \mathcal{U}$. Then $L(N+E)=$ $L(N)+L(E)=L(N)+L^{2}(E) \geqslant L(N)+L(F)$, the inequality is seen by the fact that $L$ is idempotent and $F \leqslant L(E)$. Thus, $L(N+E) \geqslant L(N+F)$, and hence $L(N+E) \in \mathcal{U}$, contradicting that $L$ strongly preserves $\mathcal{U}$. Thus, $L(E)=E$.

Now, suppose that $T(E)=T(F)$ for two cells $E$ and $F$. Then, $L(E)=T^{q}(E)=$ $T^{q}(F)=L(F)$, and from the above, $E=F$. That is, $T$ is injective on the set of cells. Since $\mathcal{E}$ is finite, $T$ is bijective on $\mathcal{E}$ (and hence is bijective on $\mathcal{M}_{m, n}(\mathbb{B})$ ).

Definition 3.3. A two-claw in a bipartite graph is a pair of edges incident with one vertex. In $\mathcal{M}_{m, n}(\mathbb{B})$, a two-claw matrix is a sum of two cells, the ones are both in the same row or column. And a matrix $A$ with all its $k=\#(A)$ nonzero entries in one row or column is called a $k$-claw.

Lemma 3.4. Let $T: \mathcal{M}_{m, n}(\mathbb{B}) \rightarrow \mathcal{M}_{m, n}(\mathbb{B})$ be a Boolean linear operator. If $T$ is bijective on the set of cells and $T$ preserves two-claws, then $T$ is a $(P, Q)$-operator.

$\operatorname{Proof}$. If $T$ preserves two-claws and is bijective, then $T$ preserves $k$-claws for all $k$. That is, $T$ preserves lines. By Lemma 2.6, $T$ is a $(P, Q)$-operator. 
Theorem 3.5. Let $T: \mathcal{M}_{m, n}(\mathbb{B}) \rightarrow \mathcal{M}_{m, n}(\mathbb{B})$ be a Boolean linear operator and let $\mathcal{U}$ be an upper ideal that separates cells. If $T$ strongly preserves $\mathcal{U}$ and $T$ preserves two-claws, then $T$ is a $(P, Q)$-operator.

P r o of. This is immediate by applying Lemma 3.2 to Lemma 3.4.

Lemma 3.6. Let $\mathcal{F}$ be a family of matrices in $\mathcal{M}_{m, n}(\mathbb{B})$ such that $O \notin \mathcal{F}$ and $J \notin \mathcal{F}$. Then we have the following assertions:

(a) $\mathcal{U}(\mathcal{F})$ is an upper ideal.

(b) If $T$ strongly preserves $\mathcal{F}$, then $T$ strongly preserves $\mathcal{U}(\mathcal{F})$.

(c) If $\mathcal{F}$ is bigraphical, then $\mathcal{U}(\mathcal{F})$ is bigraphical.

P r o of. (a) It follows from the definition of $\mathcal{U}(\mathcal{F})$.

(b) If $A \notin \mathcal{U}(\mathcal{F})$, then there is some $N$ such that $A+N \in \mathcal{F}$. Then $T(A)+T(N)=$ $T(A+N) \in \mathcal{F}$ and hence $T(A) \notin \mathcal{U}(\mathcal{F})$.

For the converse implication, let $L=T^{q}$ be idempotent (as in Lemma 3.2). Suppose that $T(A) \notin \mathcal{U}(\mathcal{F})$. Then $T(A)+Y \in \mathcal{F}$ for some $Y$. Let $Z=T^{q-1}(Y)$. Then $T^{q-1}(T(A)+Y)=L(A)+Z \in \mathcal{F}$. But then $L(A+Z)=L(A)+L(Z)=$ $L^{2}(A)+L(Z)=L(L(A)+Z) \in \mathcal{F}$. Thus, $A+Z \in \mathcal{F}$ since L strongly preserve $\mathcal{F}$. That is, $A \notin \mathcal{U}(\mathcal{F})$.

(c) It follows from the fact that $\mathcal{F}$ is bigraphical, and if $A \in \mathcal{F}$ then $P A Q \in \mathcal{F}$ for any permutation matrices $P$ and $Q$.

Lemma 3.7. Let $T: \mathcal{M}_{m, n}(\mathbb{B}) \rightarrow \mathcal{M}_{m, n}(\mathbb{B})$ be a bijective Boolean linear operator that strongly preserves isolation number $k$. Then $T$ preserves two-claws.

P r o of. If $k=1,2$ then $T$ is a $(P, Q)$-operator by Lemma 3.1 and the fact that if $T$ strongly preserves isolation number 1 it preserves isolation number 2 , and if it strongly preserves isolation number 2 it preserves isolation number 1 . Thus, suppose that $k \geqslant 3$.

Since $T$ is bijective and $\mathcal{M}_{m, n}(\mathbb{B})$ is finite, it follows, because of the Boolean arithmetic, that $T$ is bijective on the set of cells. Suppose that $E+F$ is a two claw and $T(E+F)$ is not. Then $T(E+F)$ is a matrix containing exactly two ones which are not collinear. Without loss of generality, we may assume that $T(E+F)=E_{1,1}+E_{2,2}$. Let $E_{3}, \ldots, E_{k}$ be cells such that $T\left(E_{i}\right)=E_{i, i}, i=3, \ldots, k$. Since $E+F$ is a twoclaw, $\iota(E+F)=1$. Thus, $\iota\left(E+F+E_{3}+\ldots+E_{k}\right)<k$. Since $T$ strongly preserves isolation number $k, \iota\left(T\left(E+F+E_{3}+\ldots+E_{k}\right)\right)<k$, but $T\left(E+F+E_{3}+\ldots+E_{k}\right)=$ $E_{1,1}+E_{2,2}+E_{3,3}+\ldots+E_{k, k}$ which has isolation number $k$, a contradiction. Thus, $T$ preserves two-claws.

Our main theorem is: 
Theorem 3.8. Let $T: \mathcal{M}_{m, n}(\mathbb{B}) \rightarrow \mathcal{M}_{m, n}(\mathbb{B})$ be a Boolean linear operator. Then $T$ strongly preserves isolation number $k$ for any $1 \leqslant k \leqslant \min \{m, n\}$ if and only if $T$ is a $(P, Q)$-operator.

Proof. If $T$ is a $(P, Q)$-operator, then clearly, $T$ strongly preserves isolation number $k$.

Now, suppose that $T$ strongly preserves isolation number $k$. If $k=1,2$, then $T$ is a $(P, Q)$-operator as observed in the proof of Lemma 3.7. Thus suppose that $k \geqslant 3$.

Let $\mathcal{F}_{k}$ be the set of all matrices in $\mathcal{M}_{m, n}(\mathbb{B})$ with isolation number $k$. So $T$ strongly preserves $\mathcal{F}_{k}$. By Lemma $3.6(\mathrm{~b}), T$ strongly preserves $\mathcal{U}\left(\mathcal{F}_{k}\right)$. By Lemma 2.5, $\mathcal{U}\left(\mathcal{F}_{k}\right)$ separates cells. By Lemma $3.2, T$ is bijective on the cells, and by Lemma 3.7, $T$ preserves two-claws. Therefore the theorem now follows by Theorem 3.5 .

Thus we have obtained a characterization of linear operators that preserve the isolation number of Boolean matrices.

\section{References}

[1] L. B. Beasley: Isolation number versus Boolean rank. Linear Algebra Appl. 436 (2012), 3469-3474.

[2] L. B. Beasley, C.-K. Li, S. Pierce: Miscellaneous preserver problems. A survey of linear preserver problems. Linear Multilinear Algebra 33 (1992), 109-119.

[3] L. B. Beasley, N. J. Pullman: Linear operators that strongly preserve upper ideals of matrices. Proceedings of the Twenty-Third Southeastern International Conference on Combinatorics, Graph Theory, and Computing, Florida Atlantic University, Boca Raton, USA (F. Hoffman et al., eds.). Congr. Numerantium. 88, Utilitas Mathematica Publishing, Winnipeg, 1992, pp. 229-243.

[4] L. B. Beasley, N. J. Pullman: Boolean-rank-preserving operators and Boolean-rank-1 spaces. Linear Algebra Appl. 59 (1984), 55-77.

[5] D. de Caen, D. A. Gregory, N. J.Pullman: The Boolean rank of zero-one matrices. Proceedings of the Third Caribbean Conference on Combinatorics and Computing, Bridgetown, 1981 (C. C. Cadogan, ed.). Univ. West Indies, Cave Hill Campus, Barbados, 1981, pp. 169-173.

[6] K.-T.Kang, S.-Z.Song, L. B. Beasley: Linear preservers of term ranks of matrices over semirings. Linear Algebra Appl. 436 (2012), 1850-1862.

[7] K.H.Kim: Boolean Matrix Theory and Applications. Monographs and Textbooks in Pure and Applied Mathematics 70, Marcel Dekker, New York, 1982.

[8] S.-Z. Song: Linear operators that preserve column rank of Boolean matrices. Proc. Am. Math. Soc. 119 (1993), 1085-1088.

Authors' addresses: LeR oy B. Beasley, Department of Mathematics and Statistics, Utah State University, 3900 Old Main Hill, Logan, Utah 84322-3900, United States of America, e-mail: leroy.b.beasley@usu.edu; S eok-Zun Song (corresponding author), Department of Mathematics, Jeju National University, 102 Jejudaehak-ro, Jeju-si, Jeju 690-756, Korea, e-mail: szsong@jejunu.ac.kr; Young B a e Ju n, Department of Mathematics Education, Gyeongsang National University, Jinnyangho-ro 369beon-gil, Jinju-si, Gyeongsangnam-do, Chinju 660-701, Korea, e-mail: ybjun@gsnu.ac.kr. 